\title{
Universiteit
}

Leiden

The Netherlands

\section{Diffraction-limited high-finesse optical cavities}

Kleckner, D.; Irvine, W.T.M.; Oemrawsingh, S.S.R.; Bouwmeester, D.

\section{Citation}

Kleckner, D., Irvine, W. T. M., Oemrawsingh, S. S. R., \& Bouwmeester, D. (2010). Diffractionlimited high-finesse optical cavities. Physical Review A, 81, 043814.

doi:10.1103/PhysRevA.81.043814

Version: $\quad$ Not Applicable (or Unknown)

License: $\quad$ Leiden University Non-exclusive license

Downloaded from: https://hdl.handle.net/1887/58540

Note: To cite this publication please use the final published version (if applicable). 


\title{
Diffraction-limited high-finesse optical cavities
}

\author{
Dustin Kleckner, ${ }^{1}$ William T. M. Irvine, ${ }^{2}$ Sumant S. R. Oemrawsingh, ${ }^{3}$ and Dirk Bouwmeester ${ }^{1,3}$ \\ ${ }^{1}$ Department of Physics, University of California, Santa Barbara \\ ${ }^{2}$ Department of Physics, New York University \\ ${ }^{3}$ Huygens Laboratory, Universiteit Leiden
}

(Received 29 October 2009; revised manuscript received 2 February 2010; published 15 April 2010)

\begin{abstract}
High-quality optical cavities with wavelength-sized end mirrors are important to the growing field of micro-optomechanical systems. We present a versatile method for calculating the modes of diffraction limited optical cavities and show that it can be used to determine the effect of a wide variety of cavity geometries and imperfections. Additionally, we show these calculations agree remarkably well with FDTD simulations for wavelength-sized optical modes, even though our method is based on the paraxial approximation.
\end{abstract}

DOI: 10.1103/PhysRevA.81.043814

PACS number(s): 42.25.Fx, 42.15.Eq, 42.50.Wk, 42.60.Da

\section{INTRODUCTION}

Optical cavities play a central role in devices and experiments ranging from lasers to cavity-enhanced atomic emission to interferometric gravitational wave detection. Recently a strong interest in cavities with small mirrors has been stimulated by progress in optomechanical systems [1], which could be used for probing fundamental questions in quantum mechanics and for high-sensitivity metrology in general. Proposed experiments include ground-state cooling [2-4], quantum superpositions $[5,6]$, and many others, all realized in the relatively massive mechanical degree of freedom of a micro-optomechanical system. These experiments rely on the excitation of mechanical resonators by radiation pressure; this can be implemented using an optical cavity with one microscopic end mirror attached to a micromechanical resonator. Because the optomechanical interaction is maximized when the mass of the microscopic end mirror is as small as possible and the optical finesse is as high as possible, a detailed understanding of diffraction induced cavity loss is required to optimize the system.

We present a method for calculating the mode structure and losses of diffraction limited high-finesse cavities, based on representing the optical mode as a superposition of the optical modes of a cavity with infinitely sized mirrors. This method is a significant improvement over the canonical diffraction kernel approach $[7,8]$, which is not suited to accurate simulations of very-low-loss cavities. A rudimentary calculation of this type was used previously by the authors in the context of an optomechanical system [9] and a related method was developed independently by Klaassen et al. to characterize cavities with chaotic mode structures [10]. Using this method, it is possible to calculate the effects of a wide number of imperfections, such as finite mirror size, defocusing, wavefront error, or even removal of sections of the mirror (Fig. 1) and to consider the advantages of different cavity geometries.

\section{CALCULATION METHOD}

Making use of Dirac notation, we begin by expressing the optical field, $|\Psi\rangle$, as a superposition of the modes of a cavity with infinitely sized mirrors, $\left|\psi_{s}\right\rangle$, or $|\Psi\rangle=\sum_{s} C_{s}\left|\psi_{s}\right\rangle$. We are interested in the eigenmodes of the optical cavity, given by:

$$
\gamma_{i}\left|\Psi_{i}\right\rangle=\mathbb{M}\left|\Psi_{i}\right\rangle,
$$

where $\mathbb{M}$ is the "mode-mixing operator," which gives the effect on $|\Psi\rangle$ of a round trip in the cavity. For a cavity with perfectly reflecting infinite-size mirrors, this matrix would be diagonal. The eigenvalues of the system, $\gamma_{i}$, give the field amplitude change per round trip of the corresponding eigenmode, $\left|\Psi_{i}\right\rangle$.

The problem is now reduced to choosing an convenient set of basis states and calculating the elements of the mixing operator in this basis. Although in principle we need not do so, working in the paraxial approximation greatly simplifies the calculation. This also allows us to characterize many cavity geometries via a small number of easily computed quantities. For optical cavities with radial symmetry, a convenient set of basis states is provided by Laguerre-Gaussian modes:

$$
\begin{gathered}
\psi_{n, m}^{ \pm}(\rho, \phi, \zeta)=N \rho^{|m|} L_{n}^{|m|}\left(2 \rho^{2}\right) e^{-\rho^{2} \pm i \theta(\rho, \zeta)+i m \phi} \\
\theta(\rho, \zeta)=-\zeta \rho^{2}+(2 n+|m|+1) \tan ^{-1} \zeta
\end{gathered}
$$

where $\rho=r / w(\zeta)$ and $\zeta=z / z_{0}$ are dimensionless radial and axial position coordinates, $w(\zeta)=w_{0} \sqrt{1+\zeta^{2}}$ is the mode radius, $z_{0}=k w_{0}^{2} / 2$ is the Rayleigh range, $n$ is the radial mode number, $m$ is the helicity $(|m| \leqslant n)$, and $L_{n}^{|m|}$ is a generalized Laguerre polynomial. The \pm indicates the direction of propagation and $N_{n, m}=\sqrt{\frac{2^{|m|+1} n !}{\pi(n+|m|) !}}$ is the normalization constant that ensures $\iint \rho d \rho d \phi|\psi(\zeta)|^{2}=1$. The longitudinal phase shift of the traveling electromagnetic field, $\exp [ \pm i k z]$, has been omitted and will be treated separately. The paraxial approximation should be valid as long as the waist of the cavity mode is larger than a wavelength.

We label the two end mirrors of the cavity A and B, each of which has a corresponding radius $r_{a(b)}$, radius of curvature $R_{a(b)}$ and location along the axis of symmetry $z_{a(b)}$, which is defined relative to the mode waist (Fig. 1). We then split the mode-mixing matrix in to two pieces, one for each end mirror. The elements of these matrices are given by mode overlap integrals taken over the finite extent of the mirrors:

$$
\mathbb{A}_{s, t}=\left.\int_{0}^{\rho_{a}} \int_{0}^{2 \pi} \rho d \rho d \phi \psi_{s}^{+} \psi_{t}^{-*} e^{-2 i k \Delta_{a}(\rho, \phi)}\right|_{\zeta=\frac{z a}{z_{0}}}
$$



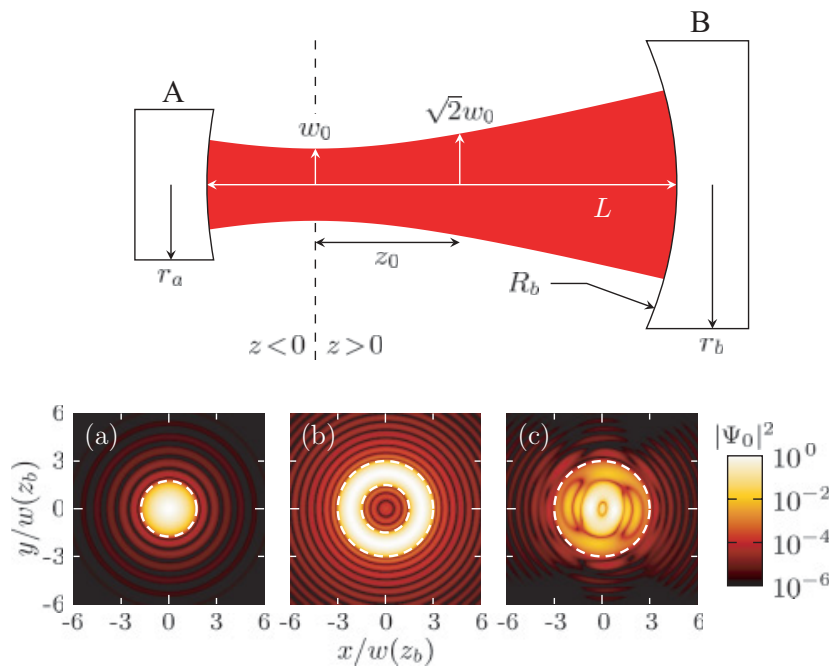

FIG. 1. (Color online) (Top) A diagram of the cavity configuration. (Bottom) An example of the effect on mode profile for several types of mirror imperfection. The profile of the fundamental mode is shown on mirror B for a cavity configuration given by Eq. (8) with $\zeta_{b} \rightarrow \infty$ and the dashed lines indicate the mirror edges. In general, the shape of the fundamental mode is found to deviate from a Gaussian in a way that minimizes loss. (a) A heavily diffraction limited cavity with $\alpha=r_{a} / w_{0}=r_{b} / w\left(z_{b}\right)=1.75$. (b) A region of radius $r_{b} / 2$ is removed from the center of mirror $\mathrm{B}$ (with $\alpha=3$ ). (c) Wavefront error is added to mirror $\mathrm{B}\left[\alpha=3, v_{r}=3\right.$ and $\sigma=$ $10^{-2} \lambda$; see Eq. (12)].

$$
\begin{gathered}
\mathbb{B}_{s, t}=\left.\int_{0}^{\rho_{b}} \int_{0}^{2 \pi} \rho d \rho d \phi \psi_{s}^{-} \psi_{t}^{+*} e^{+2 i k \Delta_{b}(\rho, \phi)}\right|_{\zeta=\frac{z_{b}}{z_{0}}} \\
\mathbb{M}=\exp [2 i k L] \mathbb{A} \times \mathbb{B},
\end{gathered}
$$

where $s$ and $t$ refer to one of the basis states and the upper bound to the integration over $\rho$ is given by $\rho_{a(b)}=$ $r_{a(b)} / w\left(\zeta_{a(b)}\right)$; the basis states will frequently be defined such that $\rho_{a}=\rho_{b}=\alpha$, where $\alpha$ is the ratio of mirror to mode size and is the same on both mirrors.

The $\Delta_{a(b)}$ term represents the deviation of each end mirror from planar - the deviation of a mirror with radius of curvature $R$ it is given by $\Delta(r) \cong \frac{r^{2}}{2 R}$. In the equation for the round trip mixing matrix, $\mathbb{M}$, we have added the overall length induced phase shift which we omitted in the definition of the basis states.

From Eq. (2), it can be shown that the radius of curvature of the mode wavefront is given by $R_{\psi}(\zeta)=z_{0}\left(\zeta+\zeta^{-1}\right)$. If this curvature is matched to the curvature of the mirror there will be no overall radial phase shift in the mixing integral; we will refer to this case as a cavity which is "in focus." Note that for a radially symmetric cavity, the $\phi$ integral is trivial, reducing to a Kronecker delta in $m$. This means helicity is preserved in these cavities, and modes with different values of $m$ can be calculated independently.

In principle, the mixing matrix $\mathbb{M}$ acts on an infinitedimensional mode space and its eigenmodes are exact solutions. Using a finite set of modes produces a perturbative solution; in this case the accuracy is dramatically improved if the basis states match the true fundamental modes of the cavity as closely as possible. When using the Laguerre-Gaussian modes, the basis states are determined by the (arbitrary) location of the $z=0$ plane relative to the mirrors and size of the mode waist, $w_{0}$, where any set of values will create an infinite set of orthogonal modes. As a rule of thumb, a sufficiently accurate choice can be made by choosing the basis which maximizes $\left|\mathbb{M}_{0,0}\right|$. For simple geometries, the choice of basis states is readily apparent, but for more complicated cases it is often more convenient to optimize them numerically.

The power loss per round trip of each mode is given by $\delta_{i}=1-\left|\gamma_{i}\right|^{2}$. Although a mode is only in resonance when $\gamma_{i}$ is real and positive, the phase shift can be corrected by a small offset to $k$, which will have negligible impact on the mode provided $k L \gg 1$. In this sense the complex phase of $\gamma_{i}$ gives the relative detuning of the different modes, which may be useful in analyzing the modes of real optical cavities.

Since we are interested in the good cavity limit, we will take the optical finesse to be $F_{i} \cong 2 \pi / \delta_{i}$. We ignore any losses due to the imperfect bulk reflectivity of the mirror, which would simply multiply all the elements of $\mathbb{M}$ by a constant. The resulting reduction in finesse is given by:

$$
\frac{1}{F_{i}}=\frac{1}{F_{M, i}}+\frac{1}{F_{R}}=\frac{1}{F_{M, i}}+\frac{1-R}{\pi},
$$

where $F_{M, i}$ is the finesse calculated by a mode-mixing calculation with perfect mirrors, $F_{R}=\pi /(1-R)$ is the finesse limited by the bulk reflectivity alone, and $R$ is the bulk reflectivity, assumed to be identical for the two mirrors.

Typically, simulating modes up to $n=15$ is accurate to several percent. In practice, we compute the integrals up to $n=$ 30 numerically using Romberg's method with a 1025 point array of double precision floating points values. This is good enough to accurately simulate cavities up to at least $F \lesssim 10^{10}$, limited by the floating point precision. Note that this limit is considerably better than the experimental limit for real cavities imposed by mirror reflectivity, which is of order $F \lesssim 10^{6}$ in the visible to near-infrared regime [11].

\section{CAVITY LENGTH}

We now demonstrate the utility of this method by using it on a variety of cavity geometries relevant to real experiments. First, to determine the effect of cavity length, we consider an "in focus" system where we fix one mirror at the mode waist:

$$
\begin{array}{ccc}
r_{a}=\rho_{a} w_{0} & z_{a}=0 & R_{a}=\infty \\
r_{b}=\rho_{b} w\left(\zeta_{b}\right) & z_{b}=L=\zeta_{b} z_{0} & R_{b}=R_{\psi}\left(\zeta_{b}\right) .
\end{array}
$$

Setting $\rho_{a}=\rho_{b}=\alpha$ implicitly chooses the waist size, $w_{0}$, which maximizes $\left|\mathbb{M}_{0,0}\right|$, and so corresponds to the optimal basis state choice for the calculation. For such a cavity, the mixing matrices are identical apart from the Gouy phase, $(2 n+$ $|m|+1) \tan ^{-1} \zeta_{b}$. This is expected; in this formalism, the Gouy phase completely describes the difference between the nearfield $\left(z \ll z_{0}\right)$ and far-field regime $\left(z \gg z_{0}\right)$.

The plot of the finesse of the first several modes as a function of dimensionless length, $\zeta_{b}$, is shownin Fig. 2 for $\alpha=2.5$, along with the finesse that would be expected for a unmodified Laguerre-Gaussian mode (calculated from the self-overlap of single modes). Whenever a mode becomes resonant with a higher-order mode (i.e., $\gamma$ has the same complex phase for both modes), we find a strong enhancement 

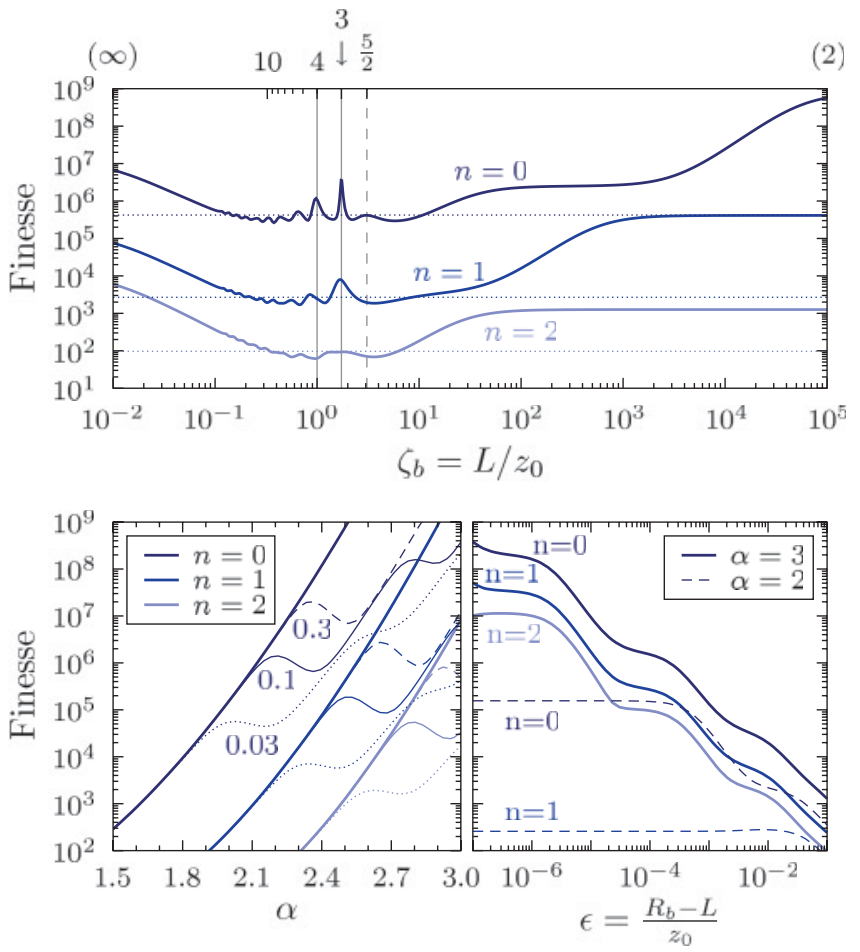

FIG. 2. (Color online) (Top) The finesse of the first several zero helicity modes as a function of cavity length. The dotted lines show the finesse expected for a simple Laguerre-Gaussian mode, which does not depend on the cavity length. The cavity geometry is given by Eq. (8) with $\alpha=2.5$. The top axis shows the location of intermode resonances as given by $j=\pi / \tan \zeta_{b}$. (Bottom left) The thick lines show the finesse as a function of $\alpha$ for a cavity with $\zeta_{b} \rightarrow \infty$. The dotted, thin, and dashed lines correspond to finite length cavities with $L=10^{5} \lambda$ and $r_{b} / L=0.03,0.1$, and 0.3 , respectively. (Bottom right) The finesse of a defocused infinite-length cavity as a function of the dimensionless defocusing parameter $\epsilon$.

of the finesse. This effect is analogous to a mode anticrossing in a coupled oscillator system; it is only when the two modes are in resonance that significant mixing is possible, which will generally increase finesse of the lower-order mode. Conversely, if two modes are just out of resonance the mode-mixing process is frustrated, in some cases reducing the finesse slightly below what would be expected for a simple Laguerre-Gaussian mode. For this cavity geometry, a resonance occurs whenever $j \tan ^{-1} \zeta_{b}=\pi$, where $j$ is an integer or rational fraction. For very long cavities $\left(L \gg z_{0}\right)$, $j \rightarrow 2$ and the finesse saturates at a dramatically increased value. Similarly for very short cavities, $\left(L \ll z_{0}\right)$, the Gouy shift goes to 0 and all the modes become nearly resonant, again increasing the finesse.

\section{LONG CAVITIES, DEFOCUSING, AND REAL MIRRORS}

The cavities used in optomechanical systems are generally composed of one small flat mirror located at the center of radius of curvature of a much larger concave mirror. This cavity, assuming it is in focus, is of the same form as Eq. (8), but in the limit $L \gg z_{0}$ and hence $R_{b} \rightarrow L$. In this case $\alpha$ is given by:

$$
\alpha=\sqrt{\frac{\pi r_{a} r_{b}}{\lambda L}}
$$

which can be determined from Eq. (2). The finesse as a function of $\alpha$ for a cavity in the $L \gg z_{0}$ limit is shown in Fig. 2 .

For a real cavity, it is difficult to ensure that the smaller end mirror is exactly at the center of radius of curvature of the larger end mirror, in which case the mode wavefronts will not be perfectly matched to the end mirrors. We can calculate the effect of this defocusing by fixing $R_{b}$ while adding a small offset to $\zeta_{b}$ :

$$
\epsilon=\Delta \zeta_{b}=\frac{R_{b}-L}{z_{0}},
$$

which results in a quadratic phase shift in $\rho$ for the overlap integrals of $\mathbb{B}$. The finesse as a function of $\alpha$ and $\epsilon$ is shown in Fig. 2. We find that high finesse cavities are extremely sensitive to length errors; for realistic cavity geometries $z_{0}$ is $10-10^{3} \mu \mathrm{m}$, requiring the cavity length to be adjusted with an accuracy of $1-100 \mathrm{~nm}$ to obtain $F>10^{6}$. In practice, the loss depends only on the magnitude of $\epsilon$ and not on the sign.

Because the ultrahigh reflectivity dielectric mirrors used in low loss cavities have a penetration depth of order wavelength, this suggests these cavities might experience loss due to an effective defocusing. Although it would be difficult to calculate this effect in the formalism presented here, we can estimate the order of magnitude of this effect by calculating the angle-dependent phase shift of this type of mirror. The lowest loss mirrors are generally composed of alternating layers of $\mathrm{Ta}_{2} \mathrm{O}_{5}(n=2.1)$ and $\mathrm{SiO}_{2}(n=1.45)$, with each layer $\lambda / 4 n$ thick. Consider a dielectric mirror composed of 20 layers of each material; a cavity made from these mirrors would has a reflectivity limited finesse of slightly over $10^{6}$. The phase shift of the reflected light, $\Delta \theta$ as a function of angle of incidence, $\varphi$, can be calculated using the thin film matrix method [12] and to fourth order is given by:

$$
\Delta \theta \cong-0.794 \varphi^{2}+ \begin{cases}0.736 \varphi^{4} & (s \text { polarization }) \\ -0.355 \varphi^{4} & (p \text { polarization })\end{cases}
$$

Apart from an overall phase shift, to order $\varphi^{2}$ the penetration depth only causes an effective change of the $z$ position of the mirror, which can be trivially compensated for. The $\varphi^{4}$ term provides an uncorrectable phase shift, but the maximum practical numerical aperture $\left(r_{b} / L\right)$ for a high-quality optical cavity corresponds to $\varphi \lesssim \frac{1}{10}$. Thus the magnitude of this phase shift is less than $10^{-4}$ at the edge of the mirror. We note that in the analysis of defocusing above, the parameter $\epsilon$ corresponds to the phase shift at the characteristic radius of the fundamental mode $(\rho=1)$. By comparison, we conclude that any effect from penetration depth should be negligible for realistic cavities $\left(F \lesssim 10^{6}, \frac{r_{b}}{L} \lesssim \frac{1}{10}\right)$.

\section{ROUGHNESS AND WAVEFRONT ERROR}

We now consider the effects of mirror surface imperfections. Microroughness, which here we will take to mean roughness on scales much smaller than the mode, can be treated as an overall reduction in the mirror reflectivity. If the surface 
roughness has an rms (root mean-squared) amplitude $\sigma$, the scattering loss is given by $\delta_{R}=1-e^{-(4 \pi \sigma / \lambda)^{2}}$ [13]. The best commercially available mirrors are superpolished to a microroughness of better than $1 \AA$, allowing finesses of $10^{6}$ to be realized in the visible regime. On the other hand, these mirrors may have long-scale surface-height imperfections, known as "wavefront error," ranging in amplitude from of order $10 \mathrm{~nm}$ for conventional optics to $1 \AA$ for use in the extreme UV.

For a micro-optomechanical system, the wavefront error of the conventionally sized mirror, $\mathrm{B}$, is the most relevant. The mirror on the optomechanical system, A, is typically fabricated on a semiconductor substrate, which are sufficiently flat over scales of tens of microns. We simulate wavefront error by adding random fluctuations to the $\Delta_{b}$, in a cavity where we again assume $L \gg z_{0}$. To examine the effect of scale, we generate normally distributed random noise with a Gaussian transverse scaling function given by:

$$
\tilde{A}_{k_{r}}=\exp \left[-8\left(1-\frac{k_{r} r_{b}}{\pi v_{r}}\right)^{2}\right],
$$

where $k_{r}$ is the wave vector of a roughness transverse frequency component and $v_{r}$ is the mean roughness frequency relative to the mirror diameter and the factor 8 in the exponent gives the relative width of the noise in $k$ space-a smaller value gives randomness with a large variation in scale, while a larger value gives a result which does not possess the desired randomness. The resulting profile is normalized to the desired rms amplitude, $\sigma$. Some example computed wavefront maps are shown in Fig. 3.

With roughness on only one mirror, the optimal basis states are no longer given by $\rho_{a}=\rho_{b}$. However, for $L \gg z_{0}$, $\rho_{a} \rho_{b}=\alpha^{2}$, where $\alpha$ is given by Eq. (9) and the individual values are numerically optimized to maximize $\left|\mathbb{M}_{0,0}\right|$. Because random roughness breaks cylindrical symmetry it is necessary to do the overlap integral in two dimensions and calculate mixing between differing values of $m$. This greatly increases the computational complexity of the calculation, and so we simulate modes only for which $n, m<20$. We also note that long-scale roughness may slightly defocus the cavity; presumably in practice an experimenter would adjust the cavity

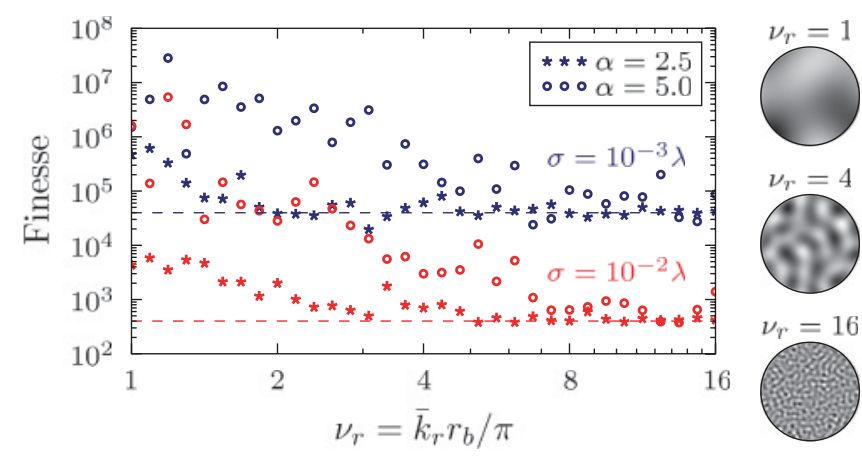

FIG. 3. (Color online) Simulated cavity finesse for the fundamental mode for rough mirrors as a function of roughness frequency, expressed relative to the mirror diameter. The large fluctuations in $F$ are due to the random nature of the roughness profile. The expected results for microroughness of the same rms amplitude are shown as dotted lines. Example roughness profiles are shown at right. length to maximize finesse. We account for this by numerically maximizing the finesse as a function of the position of mirror $\mathrm{A}$, which can be done without recalculating $\mathbb{B}$.

Results of calculations as a function of $v_{r}, \alpha$, and $\sigma$ are shown in Fig. 3. When the roughness scale is comparable to the mode size the finesse approaches a value consistent with microroughness; this is because the mode mixing couples the fundamental to much higher-order modes, which are not supported by the cavity. As $\alpha / \nu_{r}$ is increased, the effect of wavefront distortion is dramatically reduced. In this limit the wavefront error induces mixing only in the lower order modes, which are all relatively low loss. For this reason, the effects of wavefront error are largely irrelevant in cavities with two conventionally sized mirrors (where typically $\alpha \gg 1$ ). Unfortunately having a large mode size on one end of the cavity is essential to allow the smallest possible mirror on the other, posing a challenge for achieving high finesse with conventional micro-optomechanical systems.

\section{SHORT CAVITIES}

As noted previously, a finesse enhancement is also observed for very short cavities, $L \ll z_{0}$, making them an attractive candidate for optomechanical systems. For simplicity we will consider a symmetric cavity, given by:

$$
r_{a(b)}=r \quad z_{a(b)}= \pm L / 2 \quad R_{a(b)}= \pm \eta \frac{r^{2}}{\lambda} .
$$

Because of the complicated interaction between mode waist $w_{0}$ and wavefront curvature in the near-field regime, there is no clear definition of an "in focus" cavity. As a result, we characterize the mirror curvature with the dimensionless parameter $\eta=\frac{R \lambda}{r^{2}}$. If we fix the $z=0$ plane at the center of the cavity, the ratio of mirror to mode size (as determined from the definition of the Laguerre-Gaussian modes) is given by:

$$
\frac{r_{a(b)}}{w\left(z_{a(b)}\right)}=\rho_{a(b)}=\frac{r}{\sqrt{\lambda L}} \sqrt{\frac{4 \pi L z_{0}}{L^{2}+4 z_{0}^{2}}} .
$$

The optimal basis state is then determined by numerically optimizing $\left|\mathbb{M}_{0,0}\right|$ as a function of $z_{0}$. (Unlike in the long cavity case, increasing $z_{0}$, or equivalently decreasing $w_{0}$, reduces the size of the mode size on both mirrors but increases the wavefront mismatch; the resulting $\left|\mathbb{M}_{0,0}\right|$ overlap integral is complex enough that the optimum does not possess a closed form solution to the best of our knowledge.) For this cavity geometry, we find that the fundamental mode deviates more from a simple Gaussian than was previously the case, requiring calculation of modes up to $n=100$ to get accurate results. This deviation is the result of many higher modes being nearly in resonance simultaneously - this is only possible when the cavity is in the near field regime.

The results (Fig. 4) show that even a small amount of curvature on the mirrors can dramatically increase the finesse in comparison to flat mirrors. In practice, the required radius of curvature for this effect is of order $1 \mathrm{~mm}$ for $L \sim \lambda \sim 1 \mu \mathrm{m}$, far smaller than is obtainable by conventional optical polishing. Despite this, the intrinsic stress in ion-beam deposited dielectric mirrors is high enough to produce this degree of curvature if the mirrors are free standing [14]. 


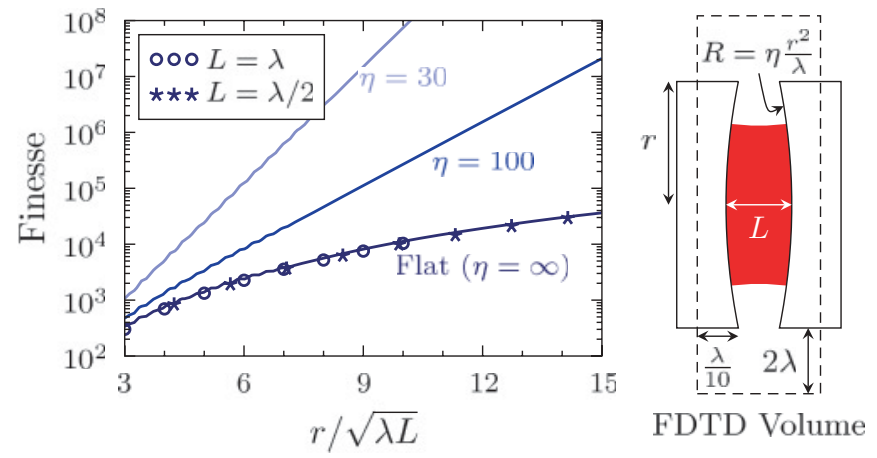

FIG. 4. (Color online) (Left) The finesse of the fundamental mode for a short cavity with symmetric mirrors. The solid lines are the results for paraxial approximations calculations including up to $n=$ 100. The fluctuations at small radii are an artifact due to the finite number of modes in the calculation. The results of FDTD simulations for flat mirrors are also shown. (Right) A diagram of the short-cavity mirror arrangement, also showing the FDTD simulated volume.

For cavities with $L \sim \lambda$ it is possible to do a full FDTD simulation of the electromagnetic field, owing to the small simulation volume. To do this we use the commercial software package LUMERICAL FDTD. ${ }^{1}$ The mirrors are represented by perfectly conducting discs of infinitesimal thickness. The simulation volume is indicated in Fig. 4, and we use a uniform simulation mesh with a spacing of $25 \mathrm{~nm}$. Due to mesh size limitations, it is only possible to simulate flat mirrors accurately. The simulated cavity is excited with a short pulse centered at the expected fundamental frequency and the resulting response of the electric field is fit to a superposition of several exponentially decaying sine waves which correspond to the different transverse modes. The results for the highest finesse modes in cavities of length $\lambda$ and $\lambda / 2$ and mirror

${ }^{1}$ From Lumerical Solutions, Inc. radii of 3-10 $\lambda$ are plotted in Fig. 4 along with results from mode-mixing calculations. The results of the simulations agree with our previous results to within 5-10\%, which is remarkable considering that the mode-mixing calculations are done in the paraxial approximation and $w_{0} \sim \lambda$. Most of the difference is likely attributable to edge effects, which can cause large angle scattering that is not properly accounted for in a calculation based on the paraxial approximation.

\section{CONCLUSION}

We have demonstrated an efficient and versatile method for calculating the mode profiles and losses of diffraction limited cavities. In particular, we have used this method to show that it is possible to achieve finesses comparable to the best conventional optical cavities when one if the end mirrors is only several $\lambda$ in size. However, such a cavity is far more sensitive to imperfections in cavity alignment and wavefront error, posing a challenge for obtaining high finesses in real micro-optomechanical systems. Alternatively, it is also possible to achieve high finesses with very short cavities, which, owing to the fact that mode size is of order $\lambda$ on both end mirrors, should not be subject to significant wavefront error. Finally, by comparison with FDTD results for very short cavities, we have shown our method works surprisingly well for wavelength sized modes, even though it is based on the paraxial approximation.

\section{ACKNOWLEDGMENTS}

The authors thank Susanna Thon for help with FDTD simulations. This work was supported by the National Science Foundation (grant PHY-0804177) and Marie-Curie EXT-CT2006-042580. Roughness calculations were performed at the California NanoSystems Institute High Performance Computing Facility, with support provided by Hewlett-Packard.
[1] I. Favero and K. Karrai, Nature Photonics 3, 201 (2009).

[2] J.-M. Courty, A. Heidmann, and M. Pinard, Eur. Phys. J. D 17, 399 (2001).

[3] I. Wilson-Rae, N. Nooshi, W. Zwerger, and T. J. Kippenberg, Phys. Rev. Lett. 99, 093901 (2007).

[4] F. Marquardt, J. P. Chen, A. A. Clerk, and S. M. Girvin, Phys. Rev. Lett. 99, 093902 (2007).

[5] S. Bose, K. Jacobs, and P. L. Knight, Phys. Rev. A 59, 3204 (1999).

[6] W. Marshall, C. Simon, R. Penrose, and D. Bouwmeester, Phys. Rev. Lett. 91, 130401 (2003).

[7] H. Kogelnik and T. Li, Appl. Opt. 5, 1550 (1966).

[8] A. Yariv, Optical Electronics (Saunders College Publishing, 1991), pp. 136-144.

[9] D. Kleckner, W. Marshall, M. J. A. de Dood, K. N. Dinyari, B.-J.
Pors, W. T. M. Irvine, and D. Bouwmeester, Phys. Rev. Lett. 96, 173901 (2006).

[10] T. Klaassen, J. de Jong, M. van Exter, and J. P. Woerdman, Opt. Lett. 30, 1959 (2005).

[11] G. Rempe, R. J. Thompson, H. J. Kimble, and R. Lalezari, Opt. Lett. 17, 363 (1992).

[12] O. Stenzel, The Physics of Thin Film Optical Spectra: an Introduction, Springer Series in Surface Sciences (Springer, 2005).

[13] P. Beckmann and A. Spizzichino, The Scattering of Electromagnetic Waves from Rough Surfaces (Pergamon, New York, 1963).

[14] J. E. Klemberg-Sapieha, J. Oberste-Berghaus, L. Martinu, R. Blacker, I. Stevenson, G. Sadkhin, D. Morton, S. McEldowney, R. Klinger, P. J. Martin et al., Appl. Opt. 43, 2670 (2004). 